\title{
FPU recurrence electromagnetic spectrum as a possible physiotherapeutic tool
}

\author{
Shmid AV, Novopashin MA, Zimina KA and Berezin AA* \\ EC-leasing Moscow, Russian Federation, Russia
}

\begin{abstract}
The idea of forced external synchronization of the heart dynamics by the canonical FPU spectrum with a purpose to lower the rate of its desynchronization in some pathological cases has been hypothesized by the authors. It was concluded that a heart being a multi resonant distributed dynamic ion containing system may be resonantly influenced by applying to it the canonical FPU electromagnetic spectrum, which can supposedly decrease the rate of desynchronization in the ECG Fourier spectrum for example in case of arrhythmia. The complex FPU recurrences found in the deep-water dynamics studies were compared with real ECG Fourier images in a sequence of time periods. For choosing the appropriate form of the external FPU canonical spectrum, the two different forms of the ECG Fourier spectra were studied: a rectangular pulse spectrum and exponential pulse spectrum. The two functions were taken to form the external synchronizing canonical FPU recurrence spectrum and were put into the right part of the equations of the previously developed mathematical model as perturbing functions. The computer study of the model simulating arrhythmia with a synchronizing perturbing part in a form of the canonical FPU recurrence spectrum changed the solutions of the model to the forms characteristic for normally functioning heart. Thus, the hypothesis has been confirmed,
\end{abstract}

\section{Introduction}

The FPU recurrence discovered during a computer simulation of dynamics in a chain of linked non- linear oscillators [1] proved to be a universal feature of distributed dynamic systems to which belong all biological systems. Differ from the Poincare recurrence, which requires the return of both amplitude and phase to their initial state [2], the FPU complex recurrence demonstrates non - identical return patterns to the initial state. The discovery of the FPU complex recurrence in such non-linear media as plasma [3], deep-water waves [2], in non-linear electrical transmission lines [4], and in the electrical activity of heart [5] in its enlarged form which was named the auto FPU recurrence, manifested omnipresence of this phenomenon in nature. The typical pattern of the FPU recurrence in the dynamics of deep-water gravity waves is shown in the Figure 1 [2].

It is possible to conclude that a heart being a multi resonant distributed dynamic system filled with electrolyte may be resonantly influenced by a canonical FPU electromagnetic spectrum, which can supposedly decrease the rate of desynchronization in the ECG Fourier spectrum for example in case of arrhythmia.

\section{Hypothesis}

Since it was previously theoretically shown that a resonant interaction between the FPU recurrences is real [6], there could be put forward a hypothesis of a possible resonant correction of the desynchronized complex FPU recurrence structure of the ECG Fourier spectra sequence in some pathological states by non-invasive application on the heart the canonical FPU recurrence electromagnetic spectrum which could diminish the rate of the ECG Fourier spectral desynchronization.

\section{Verification of the hypothesis through mathematical modeling}

The distortion of the FPU recurrence - ECG spectrum in case of arrhythmia (Figure 3) can be hypothetically forcedly synchronized in the process of a resonant interaction [6] with the canonical FPU recurrence spectrum. To check this assumption the computer modeling of the process has been carried out. For this purpose the mathematical model of the heart electrical activity within the framework of the auto FPU recurrence concept [5] has been used. The real ECG Fourier spectra analysis revealed two their extreme forms: so called the Fourier image of a rectangular impulse (Figure 4 upper left and right graphs) and the exponential impulse (Figure 4. low left and right graphs).

All mentioned above suggested canonical external synchronizing FPU recurrence spectrum generation in a form of combination of a harmonic function and an exponential function. The obtained canonical FPU functions have been put into the right part of the model equations [5] as perturbing functions. The initial model ECG solution of the coupled equations system for the case of arrhythmia and its Fourier spectrum are shown in the Figures 6 and 7.

Thus, as a result of modeling there was shown a principal possibility of the ECG Fourier spectrum forced normalization by applying a synchronizing external function in the form of the canonical FPU recurrence spectrum. Since a heart represents a distributed dynamic ionic resonator an applied electromagnetic FPU spectrum can be used as a therapeutic tool.

\section{Discussion}

The FPU recurrence looks to be an effective instrument for evaluation the heart electrical dynamics picture. Differ from the Poincare recurrence, which requires the return of both amplitude and

Correspondence to: Berezin AA, EC-leasing Moscow, Russian Federation, Russia,E-mail: artparis@mail.ru

Received: February 09, 2018; Accepted: February 15, 2018; Published: February 21,2018 

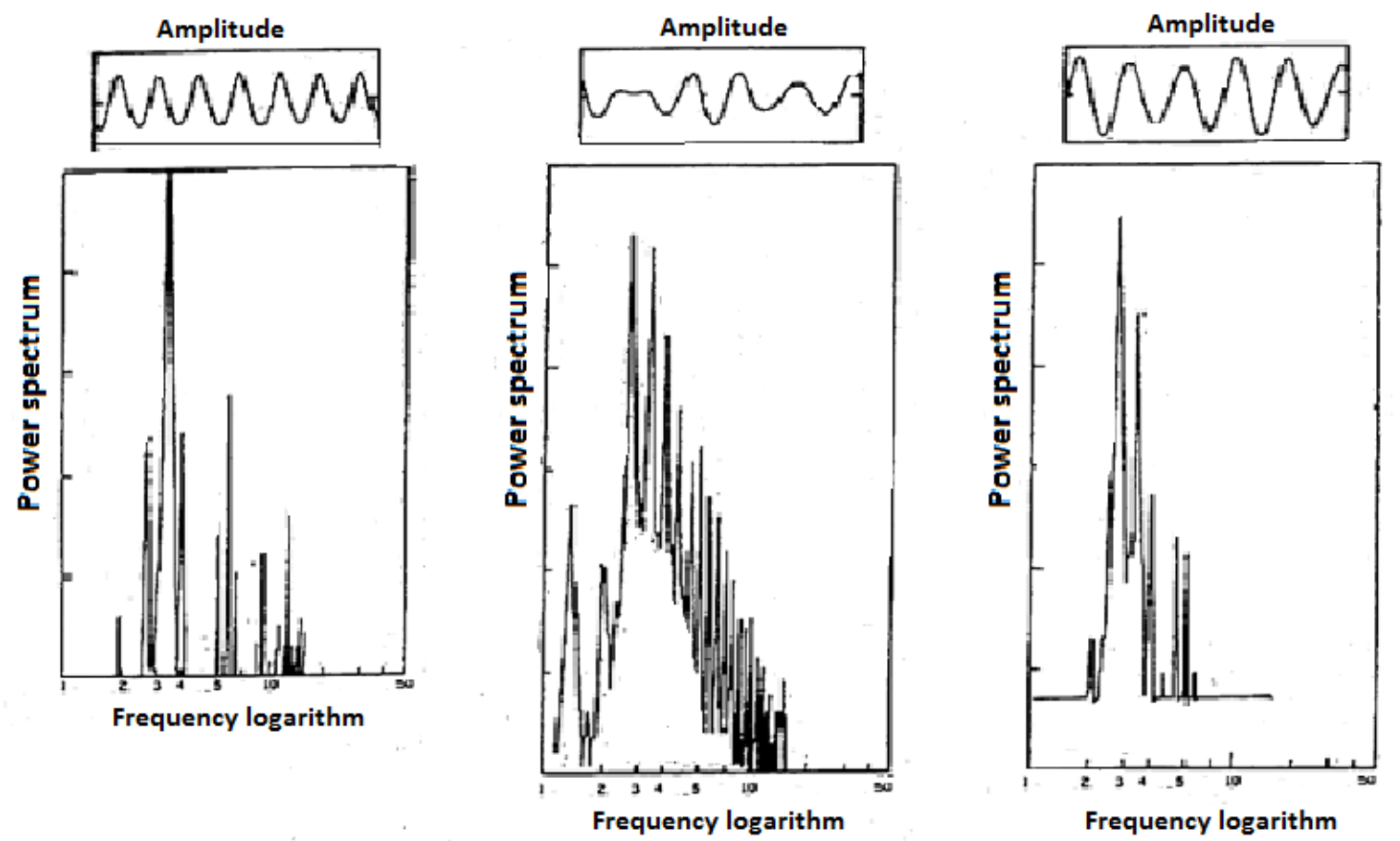

Figure 1. Complex FPU recurrence in the deep - water dynamics in a periodical sequence of observations.
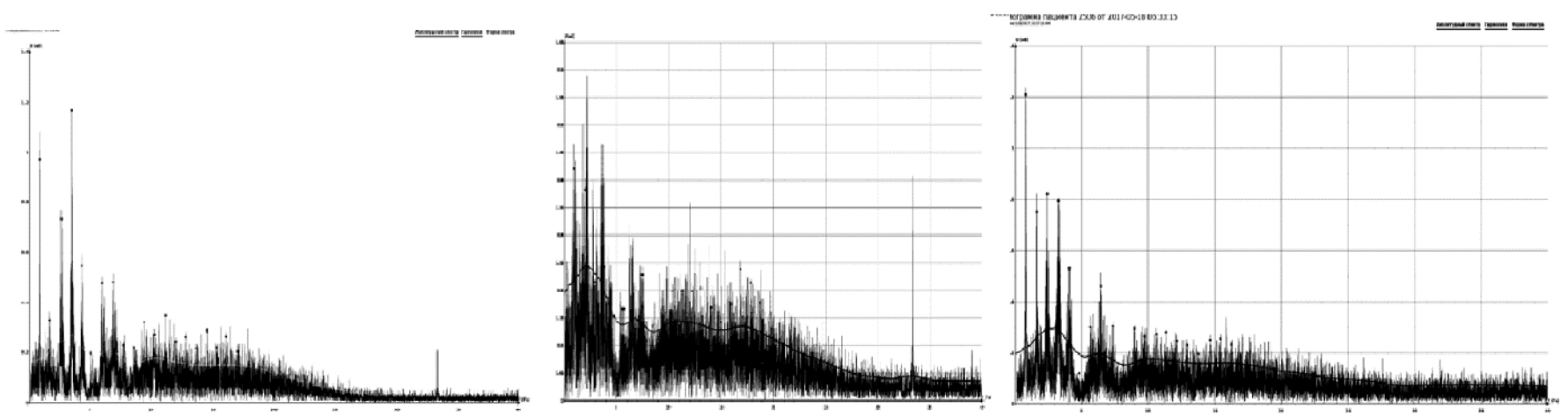

Figure 2. Complex FPU recurrence in the evolution of the Fourier spectra of the electrocardiographic data of a patient in a sequence of time. The middle graph shows desynchronization of the ECG spectrum due to dystonia. Horiz. Axis frequency Hz. Vert. Axis. mv.

Desynchronization of the ECG spectrum is more vividly expressed in case of arrhythmia as it can be seen in Figure 3.

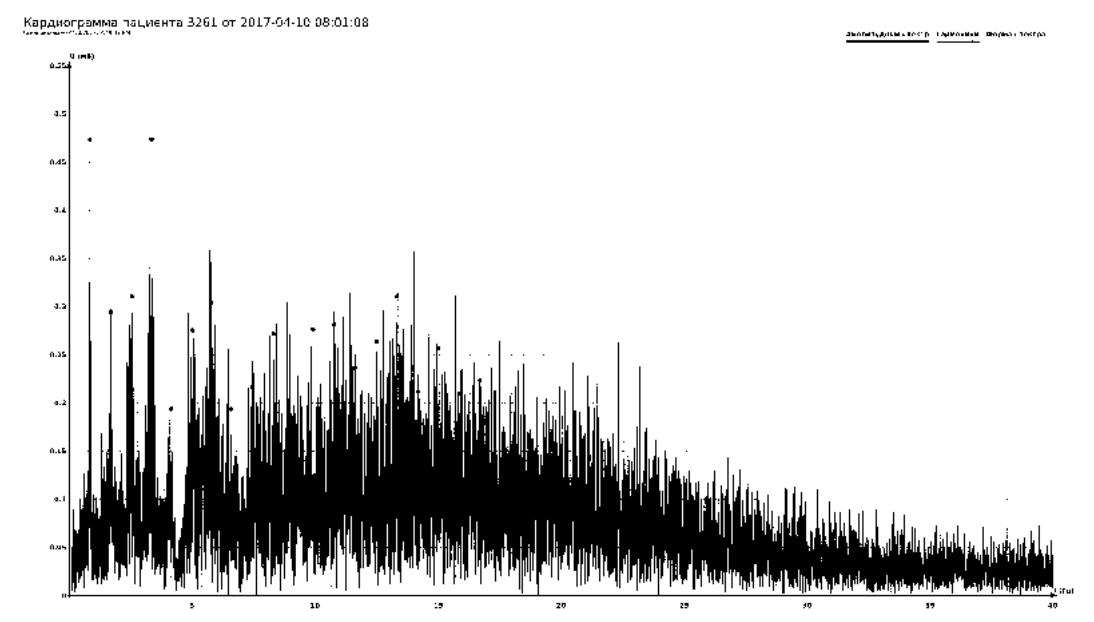

Figure 3. Fourier image of the patient's ECG with arrhythmia. Horiz. Axis frequency Hz. Vert. Axis. mv. 

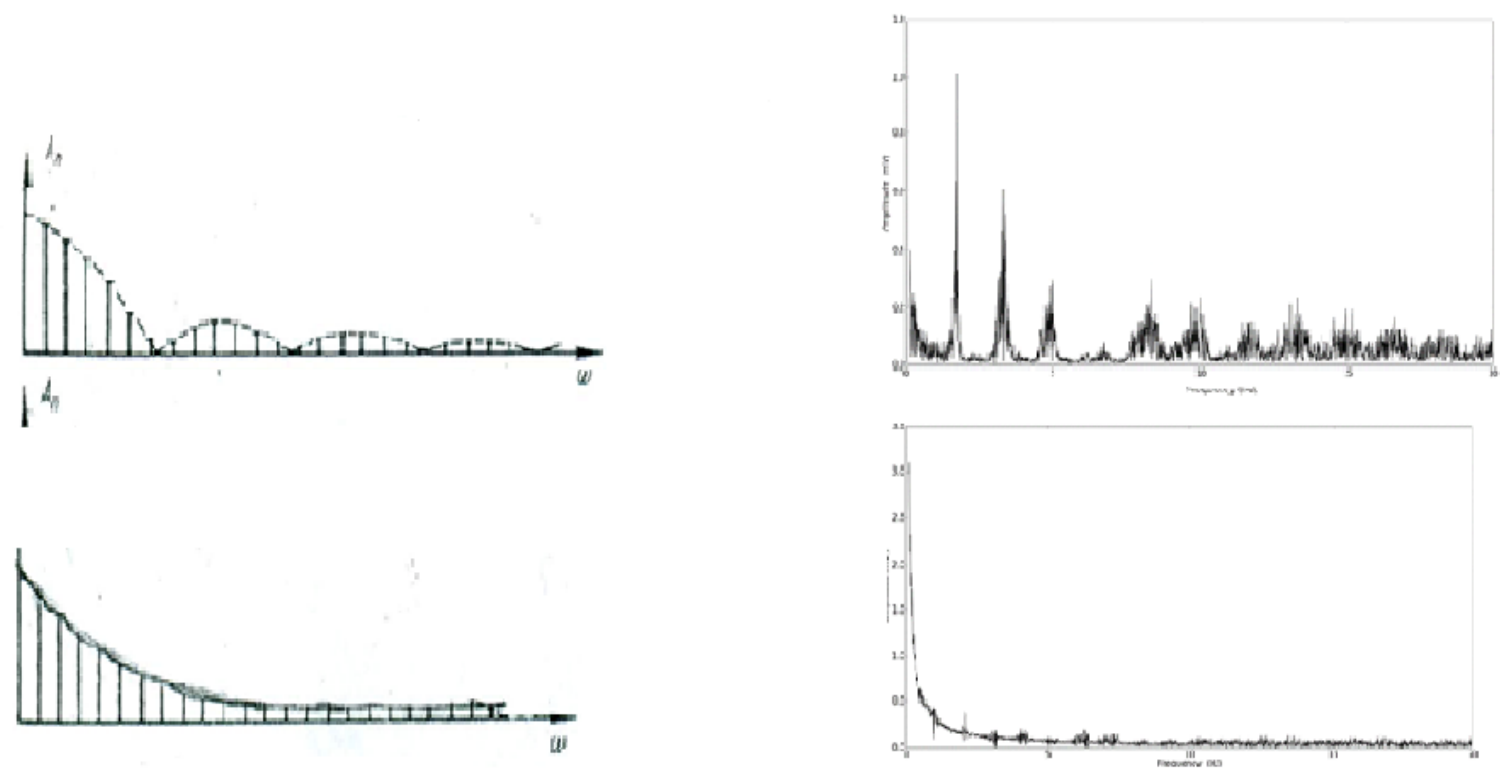

Figure 4. Fourier image of a rectangular impulse (upper left graph, conditional units) and related ECG Fourier image (upper right graph Horiz. Axis frequency Hz. Vert. Axis. mv) and the Fourier image of an exponential impulse (low left graph, conditional units) and related ECG Fourier image (low right graph Horiz. Axis frequency Hz. Vert. Axis. mv).

A regular cardiostimulator has a Fourier spectrum representing a combination of rectangular and exponential pulses Fourier images (Figure 5).

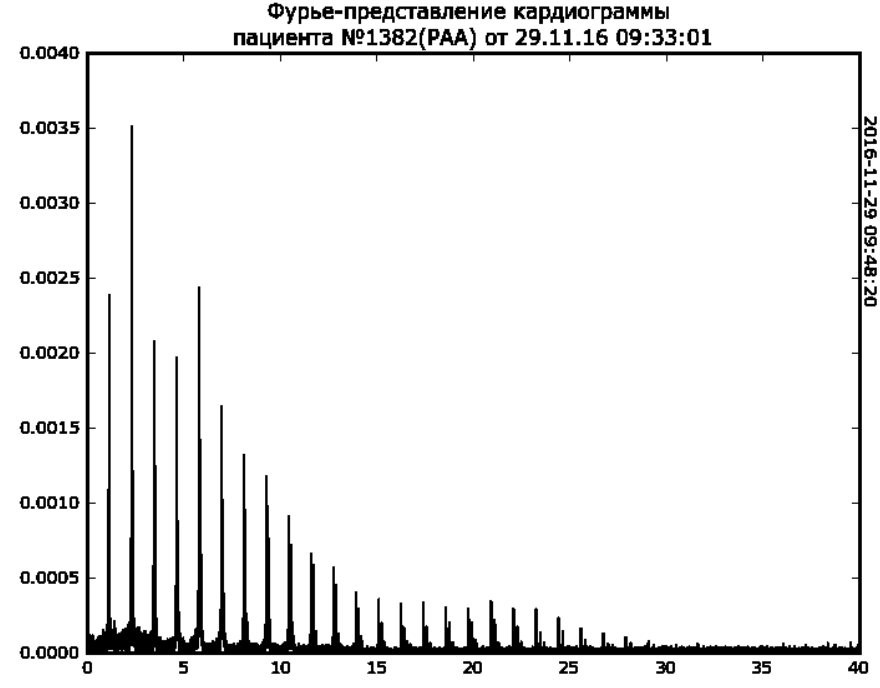

Figure 5. Fourier spectrum of the real ECG with a cardiostimulator realizing a combination of the Fourier spectra of both rectangular and exponential pulses. Horiz. Axis frequency Hz. Vert. Axis. mv.

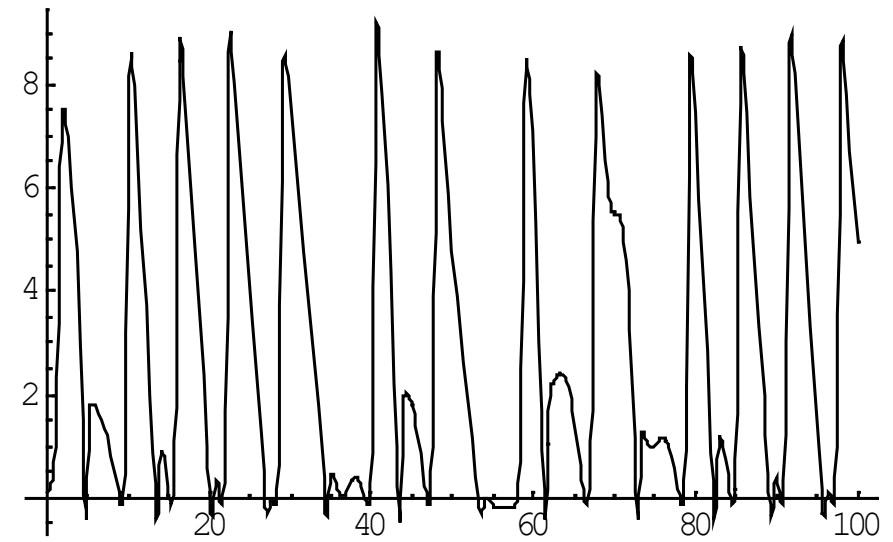

Figure 6. Model ECG solution of the mathematical model for the case of arrhythmia. Horiz.axis - frequency vert.axis - amplitude, conditional units. 


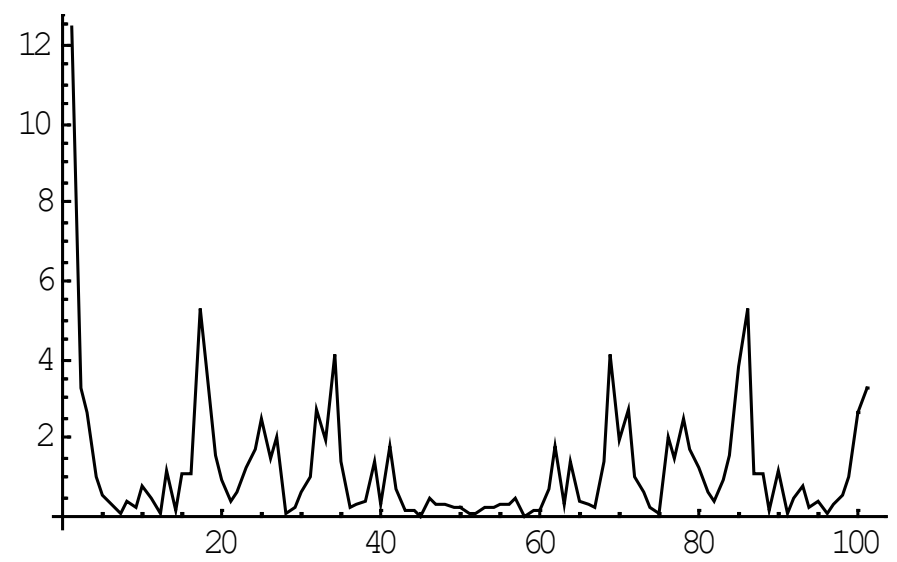

Figure 7. Fourier spectrum of the ECG arrhythmia solution of the mathematical model. Horiz.axis - frequency vert.axis - amplitude, conditional units

After applying to the model, the synchronizing function in a form of the canonical FPU spectrum the arrhythmia ECG for and its Fourier spectrum changed for normal patterns as it can be seen in Figures 8 and 9.

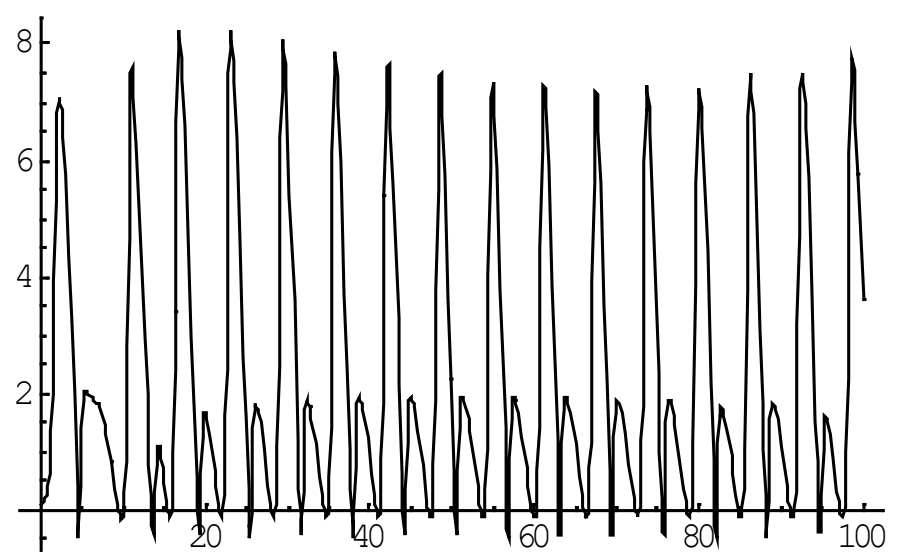

Figure 8. Normalized model ECG solution of the mathematical model after applying the spectrum of the canonical FPU recurrence. Horiz.axis - frequency vert.axis - amplitude, conditional units.

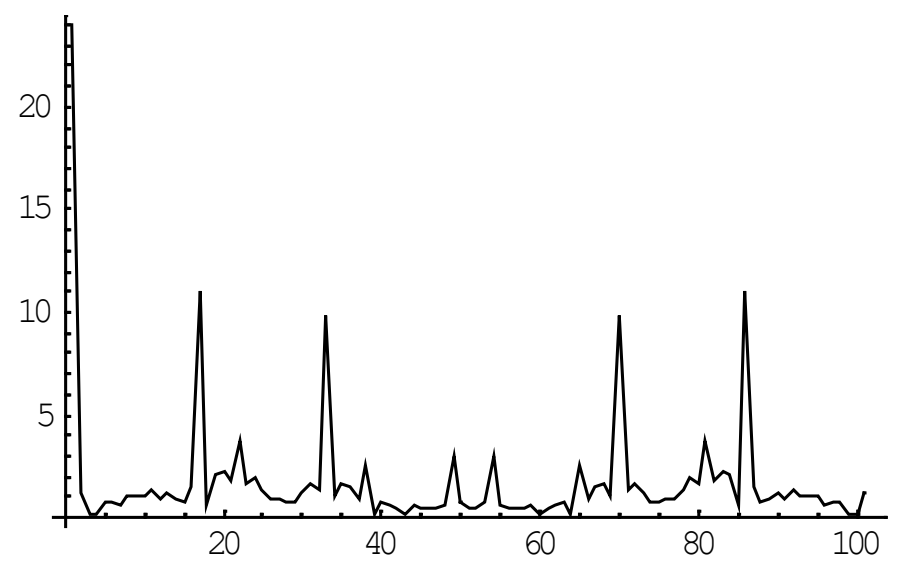

Figure 9. Fourier spectrum of the normalized ECG solution of the mathematical model after applying the spectrum of the canonical FPU recurrence. Horiz.axis - frequency vert. axis - amplitude, conditional units. phase to their initial state, the FPU complex recurrence demonstrates non - identical return patterns to the initial state. That means that FPU analysis for enough long periods of heart activity gives a possibility of prediction its behavior for the future. Moreover, it is possible to conclude that a heart being a multi resonant distributed dynamic system may be resonantly influenced by a canonical FPU electromagnetic spectrum, which can supposedly decrease the rate of desynchronization in the ECG Fourier spectrum for example in case of arrhythmia. Mathematical modeling proved the reality of this idea. After applying to the heart activity mathematical model the synchronizing external function in a form of the canonical FPU spectrum the arrhythmia ECG form as well as its Fourier spectrum structures changed to normal patterns. So the hypothesis has been confirmed.

\section{References}

1. Fermi E, Pasta J and Ulam S (1955) In: Collected Papers of E. Fermi. 2: 978.

2. Yuen HC, Lake BM (1982) Non-linear dynamics of deep-water gravity waves. $A d v$ Appl Mech 22: 67-226.

3. Ikezi H (1978) Solitons in plasmas.//in Solitons in Action. Academic Press 163-184.

4. Longren KE (1978) Solitons in non -linear electric lines. // in Solitons in Action. Academic Press $138-162$

5. Novopashin MA, Shmid AV and Berezin AA (2017) Fermi-Pasta-Ulam auto recurrence in the description of the electrical activity of the heart Med Hypotheses. [Crossref]

6. Berezin A.A (2004) Resonant interaction between the Fermi-Pasta-Ulam recurrences.// Bulletin of the Lebedev Physics Institute. FIAN 3: 13.
Copyright: C2018 Shmid AV. This is an open-access article distributed under the terms of the Creative Commons Attribution License, which permits unrestricted use, distribution, and reproduction in any medium, provided the original author and source are credited. 